\title{
Le mouton Djallonké à Kolokopé (Togo) : paramètres de reproduction des brebis et viabilité des agneaux
}

\author{
A. Missohou ${ }^{1}$ B. Bonfoh ${ }^{2}$ A.K. Kadanga ${ }^{1}$
}

\section{Mots-clés}

O vin - Mouton Djallonké - Intervalle entre parturitions - Performance de reproduction - Taille de la portée Taux de natalité - Mortalité - Togo.

\begin{abstract}
Résumé
Ce travail qui avait pour objet d'étudier les performances de reproduction des brebis et la viabilité des agneaux Djallonké au Centre d'appui technique de Kolokopé (Togo) a porté sur 163 brebis et 4503 agneaux. Les données ont été collectées de 1984 à 1994. L'âge à la première mise bas (APMB) était de $595,8 \pm 15,7$ j (19,5 mois) pour un intervalle entre mises bas (IMB) de 304 $\pm 8,2$ j (10 mois). La taille de la portée et le taux annuel de prolificité étaient respectivement de 1,31 $\pm 0,02$ et de 1,57 agneau par brebis. Le taux de mortalité avant sevrage était de 11,7 p. 100. L'APMB était significativement influencé par l'année $(p<0,001)$, la saison de naissance $(p<0,05)$ et le type d'agnelage $(p<0,05)$. Q uant à I'IMB, il était significativement affecté par l'année $(p<0,001)$, la saison $(p<0,001)$ et le type d'agnelage $(p<0,05)$. Le taux de mortalité présevrage était significativement affecté par l'année $(p<0,001)$ et le type de naissance $(p<0,001)$.
\end{abstract}

\section{INTRODUCTION}

Les pays côtiers d'Afrique de l'Ouest sont dépendants, soit des pays du Sahel, soit de l'Occident, en particulier de l'Union européenne, pour la couverture d'une bonne partie de leurs besoins en protéines d'origine animale. Les différents cycles de sécheresse qui ont sévi au Sahel au cours de ces trois dernières décennies ont cependant éprouvé les potentialités de production des pays sahéliens qui sont exportateurs de viande aujourd'hui mais pourraient devenir importateurs en l'an 2000 (6). Par ailleurs, la viande européenne, bien que subventionnée, représente un lourd fardeau pour les Etats. Ces tendances, aggravées aujourd'hui par la décote du franc CFA et la politique de réduction de la subvention à l'exportation des produits agricoles européens, ont été perçues assez tôt par certains Etats côtiers qui, forts de leur importante production fourragère, ont décidé de mettre en place des stratégies endogènes de couverture de leurs besoins en produits carnés. Le mouton Djallonké, par sa rusticité, sa trypanotolérance, la brièveté de son cycle de reproduction, et la réserve monétaire facilement mobilisable qu'il constitue en milieu paysan, a été perçu comme l'un des supports de cette ambition. Ainsi a été créé au Togo en 1982 le

1. Service de Zootechnie-Alimentation, Ecole Inter-Etats des Sciences et Médecine Vétérinaires de Dakar, BP 5077, Dakar, Sénégal

2. Projet « Petit ruminant », BP 65, Atakpamé, Togo
Centre d'appui technique de Kolokopé (CAT-K) chargé de promouvoir l'élevage de cette race. Les premiers résultats obtenus dans ce centre ont porté sur la croissance présevrage des agneaux (1) et constituent les seules données techniques publiées chez le mouton Djallonké au Togo.

Compte tenu de l'importance de la reproduction dans la productivité du cheptel, cette note avait pour objectif de préciser quelques paramètres de reproduction et de viabilité des agneaux dans le cadre du CAT-K.

\section{MATERIEL ET METHODES}

\section{Cadre}

Les présents travaux ont été effectués au CAT-K, Togo, situé dans la région des plateaux, à $12 \mathrm{~km}$ d'Anié. Cette station jouit d'un climat soudanien à saison pluvieuse s'étendant de mi-mars à mi-octobre et à saison sèche le reste de l'année. La moyenne annuelle des précipitations au cours de cette dernière décennie (1984-1994) s'est élevée à $1197 \mathrm{~mm}$ et a varié de $718 \mathrm{~mm}$ à $1842 \mathrm{~mm}$ (tableau I). Le relief est dominé par la plaine du Mono avec quelques rares dépressions. Le sol, très riche en matières organiques, est de couleur noir foncé homogène. Classé parmi les 
meilleurs sols du Togo, il porte de belles cultures de coton, d'ignames et de maïs. La végétation sur l'ensemble de la station est une savane boisée à arborée, claire à semi-dense. Le cortège ligneux est formé de Vittelaria paradoxa, Terminalia macroptera, Combretum glutinosum, Acacia sp. et Euphorbia sp. La strate herbacée est dominée par Panicum maximum et Sorghum arundinaceum.

\section{Conduite des animaux}

Les brebis dont l'effectif moyen annuel était de 800 têtes étaient élevées en troupeaux de 150 animaux environ, chaque troupeau étant placé sous la responsabilité d'un berger titulaire. En 1992, un noyau de 60 chèvres et de huit boucs ont été introduits au CAT-K. Les brebis qui avaient agnelé étaient gardées dans la bergerie pendant les 3-7 premiers jours qui ont suivi la mise bas. Les troupeaux étaient menés sur pâturages améliorés formés de Panicum, de Leucaena, de Gliricidia et de Cajanus pendant six heures par jour maximum. La supplémentation de ces animaux se faisait toute l'année avec des graines de coton à raison de 150 à $300 \mathrm{~g}$ par tête et par jour (en fonction du stade physiologique des animaux et de la valeur nutritive du pâturage) et des blocs de sels minéraux. Les jours pluvieux, les animaux étaient gardés à la bergerie où des branches de légumineuses arbustives et du foin de Panicum leur était distribués.

La reproduction se faisait par des luttes naturelles organisées avec des lignées de géniteurs tous les huit à neuf mois pendant une durée de 45 jours. Les antenaises étaient mises à la reproduction en fonction des besoins de renouvellement du cheptel, sans prise en compte réelle de leur poids vif. Le rapport mâle/femelle était environ de $1 / 20$. Le sevrage intervenait entre trois et quatre mois d'âge avec séparation des agnelles et des agneaux.

La lutte contre les principales pathologies constatées (ecthyma contagieux, adénocarcinome, œstrose, épididymite contagieuse ovine, diarrhée des jeunes...) reposait sur des méthodes thérapeutiques spécifiques et prophylactiques. Au plan prophylactique, les animaux étaient vaccinés contre la peste des petits ruminants (Tissupest $^{\mathrm{ND}}$ ) et l'épididymite contagieuse ovine (Rev1 $\left.{ }^{\mathrm{ND}}\right)$. Le déparasitage des brebis adultes se faisait au début, au milieu et à la fin de la saison des pluies. Les jeunes, quant à eux, étaient déparasités à l'âge de 1,5 mois et au sevrage. Le déparasitage était interne $\left(\right.$ Valbazen $^{\mathrm{ND}}$ ) et externe (Butox 7,5 pour on ${ }^{\mathrm{ND}}$, Taktic ${ }^{\mathrm{ND}}$ ).

\section{Analyse des données}

Les données ont porté sur 163 brebis et 4503 agneaux Djallonké et ont été collectées entre 1984 et 1994. Toutefois les données exploitables chez les brebis n'ont été disponibles qu'à partir de 1988. Un modèle linéaire à effets fixes a permis d'étudier les facteurs de variation des paramètres étudiés. Sous sa forme générale, le modèle s'écrit :

$$
\mathrm{Y}_{\mathrm{ijklm}}=\mu+\mathrm{A}_{\mathrm{i}}+\mathrm{S}_{\mathrm{j}}+\mathrm{X}_{\mathrm{k}}+\mathrm{T}_{1}+\mathrm{e}_{\mathrm{ijklm}}
$$

où :

$\mathrm{Y}_{\mathrm{ijklm}}=$ observation sur le $\mathrm{n}^{\mathrm{e}}$ animal,

$\mu=$ moyenne générale,

$\mathrm{A}_{\mathrm{i}}=$ année de naissance (1984-1994),

$\mathrm{S}_{\mathrm{j}}=$ saison de naissance (pluvieuse ou sèche),

$\mathrm{X}_{\mathrm{k}}=$ sexe des animaux (mâle ou femelle),

$\mathrm{T}_{1}=$ type de naissance des animaux (simple, double, triple ou quadruple), et

$\mathrm{e}_{\mathrm{ijklm}}=$ effet aléatoire résiduel du modèle.

Les taux de mortalité ont été calculés comme suit :

taux de mortalité à la naissance $=$ nombre de morts à la naissance/nombre de nés ;

taux de mortalité au sevrage $=$ nombre de morts entre la naissance et le sevrage/nombre de sevrés.

\section{RESU LTATS ET DISCU SSIO N}

\section{Age à la première mise bas}

Dans les conditions d'élevage du CAT-K, l'âge à la première mise bas était de 595,8 $\pm 15,7 \mathrm{j}$ (tableau II) soit 19,5 mois. Les résultats de cette étude sont comparables à ceux obtenus en station au Cameroun, au Sénégal et au Ghana (tableau III) mais sont plus faibles que ceux obtenus en milieu traditionnel. En Côte d'Ivoire, où l'âge à la première mise bas est relativement faible en station, les femelles ont été mises à la reproduction à huit mois d'âge (11).

\section{Tableau I}

Pluviométrie mensuelle au CAT-K (Togo) de 1984 à 1994 (en mm de pluie)

\begin{tabular}{|c|c|c|c|c|c|c|c|c|c|c|c|c|}
\hline & 1984 & 1985 & 1986 & 1987 & 1988 & 1989 & 1990 & 1991 & 1992 & 1993 & 1994 & Moyenne \\
\hline Janvier & - & 4,7 & 0,8 & - & 1,1 & - & 31,9 & - & - & - & 8,8 & 9,5 \\
\hline Février & - & - & 23,6 & - & 1,2 & 13,0 & 0,6 & 88,4 & 62 & 9,4 & - & 7,0 \\
\hline Mars & 124,2 & 121,2 & 71,9 & 113,3 & 89,2 & 113,9 & - & 50,3 & 97 & 105 & 79,5 & 96,6 \\
\hline Avril & 116,5 & 141,8 & 107,9 & 2,2 & 70,1 & 82,3 & 191,1 & 192,1 & 31,1 & 38,4 & 132,4 & 100,5 \\
\hline Mai & 170,0 & 98,2 & 92,8 & 205,2 & 112,3 & 9,1 & 67,1 & 304,6 & 173,6 & 143,7 & 183,3 & 141,8 \\
\hline Juin & 131,7 & 349,9 & 33,5 & 137,8 & 140,8 & 223,0 & 215,6 & 141,3 & 162,1 & 192,4 & 80,3 & 164,4 \\
\hline Juillet & 155,8 & 329,1 & 123,2 & 93,6 & 331,4 & 168,3 & 167,8 & 363,1 & 251,0 & 118,3 & 131,5 & 203,0 \\
\hline Août & 117,1 & 376,5 & 87,4 & 385,2 & 128,3 & 311,6 & 59,9 & 185,2 & 52,9 & 235,9 & 166,4 & 191,5 \\
\hline Septembre & 142,2 & 232,1 & 140,9 & 219,3 & 129,6 & 92,9 & 117,1 & 67,0 & 145,2 & 138,7 & 236 & 151,0 \\
\hline Octobre & 48,8 & 69,4 & 96,9 & 103,4 & 113,4 & 130,8 & 54,0 & 89,1 & 88,9 & 92,6 & 221,8 & 100,8 \\
\hline Novembre & 0,2 & 119,1 & 19,6 & - & - & - & 65,8 & - & 1,7 & 48,4 & 18,5 & 39,0 \\
\hline Décembre & - & - & - & - & 47,2 & 0,5 & 97,7 & 5,3 & 5,1 & 33,7 & - & 31,5 \\
\hline Total & 1006 & 1842 & 718 & 1260 & 1165 & 1145 & 1069 & 1486 & 1071 & 1156 & 1250 & 1197 \\
\hline
\end{tabular}




\section{Tableau II}

Moyennes estimées par la méthode des moindres carrés de l'âge au premier agnelage des brebis Djallonké élevées au CAT-K

\begin{tabular}{|c|c|c|}
\hline Variables & Nb. & $\begin{array}{c}\text { Age au } \mathbf{1}^{\mathrm{e}} \\
\text { agnelage (j) }\end{array}$ \\
\hline Moyenne générale & 163 & 595,8 \\
\hline \multicolumn{3}{|l|}{$\begin{array}{l}\text { Année de naissance } \\
\text { des brebis* }\end{array}$} \\
\hline 1988 & 3 & 641,7 \\
\hline 1989 & 73 & 552,5 \\
\hline 1990 & 46 & 669,2 \\
\hline 1991 & 10 & 666,5 \\
\hline 1992 & 31 & 561,8 \\
\hline \multicolumn{3}{|l|}{$\begin{array}{l}\text { Saison de naissance } \\
\text { des brebis ** }\end{array}$} \\
\hline Pluvieuse & 136 & 596,9 \\
\hline Sèche & 27 & 590,2 \\
\hline \multicolumn{3}{|l|}{$\begin{array}{l}\text { Type de naissance } \\
\text { des brebis }\end{array}$} \\
\hline Simple & 100 & 600,8 \\
\hline Double & 63 & 587,8 \\
\hline \multicolumn{3}{|l|}{ Type d'agnelage ** } \\
\hline Simple & 137 & 580,7 \\
\hline Double & 26 & 675,5 \\
\hline
\end{tabular}

L'accouplement n'étant pas contrôlé en milieu traditionnel, les différences observées entre les deux milieux pourraient être dues au fait que les brebis sont montées juste après la puberté qui, selon Jolland (1960) cité par Tuah et Baah (13), survient entre 4 et 8 mois d'âge. Pour une durée de gestation de $150 \mathrm{j}$, l'âge à la première conception était dans la présente étude de 14,5 mois, soit plus du double de l'âge à la puberté. Une prise en compte du poids vif des antenaises (il doit correspondre à 50-60 p. 100 du poids adulte (3)) au moment de leur mise en service devrait permettre d'obtenir le premier agnelage vers 13-14 mois d'âge.

L'année et la saison de naissance des brebis et le type d'agnelage ont un effet significatif sur l'âge à la première mise bas. Fall et coll. (8) n'ont pas observé d'effet significatif de ces facteurs sur cette variable. Toutefois ils ont rapporté un âge plus élevé pour les naissances doubles que pour les naissances simples, ce qui corrobore les différences observées entre les deux types de naissance dans ce travail.

\section{Intervalle entre mises bas}

L'intervalle entre mises bas (IMB) était de 304,0 \pm 8,2 j, soit environ 10 mois, et était plus élevé que ceux rapportés par d'autres auteurs (tableau III). L'IMB comprend la durée de gestation et la période de service qui est l'intervalle entre la parturition et la fécondation. La durée de la gestation étant peu variable, c'est la période de service qui a justifié l'allongement de l'IMB constaté. En l'absence de saisonnement chez les moutons tropicaux, en particulier dans les zones où l'alimentation ne constitue pas de contrainte majeure (7), Tuah et Baah (13) pensent qu'il est possible d'obtenir chez la brebis Djallonké deux naissances par an étant donné le retour des chaleurs 14 jours après la mise bas (Jolland, cité par les mêmes auteurs). L'allongement de l'IMB rapporté dans cette étude pourrait être le résultat de la longue période de repos (8-9 mois) qui sépare les périodes de monte.

Tableau III

Performances de reproduction du mouton Djallonké dans différents pays d'Afrique

\begin{tabular}{|c|c|c|c|c|c|}
\hline Pays & $\begin{array}{l}\text { Age } 1^{\mathrm{e}} \\
\text { mise bas } \\
\text { (mois) }\end{array}$ & $\begin{array}{l}\text { Intervalle } \\
\text { entre } \\
\text { mises bas } \\
\text { (mois) }\end{array}$ & Fertilité & $\begin{array}{l}\text { Taille de } \\
\text { la portée }\end{array}$ & Référence \\
\hline \multicolumn{6}{|l|}{ Station } \\
\hline Burkina Faso & & & & 1,18 & Boly et coll. (5) \\
\hline Cameroun & 16,9 & 7,93 & 96 & 1,17 & Vallerand et Branckaert (14) \\
\hline Côte d'Ivoire & 13,5 & 8 (imposé) & $94-95$ & 1,10 & Berger et G inisty (4) \\
\hline Côte d'Ivoire & 13,5 & 8 à 10 & 94,9 & 1,17 & Rombaut (11) \\
\hline Ghana & 20,9 & 10 & & 1,30 & Tuah et Bah (13) \\
\hline Sénégal & 18,8 & 10,23 & & 1,12 & Fall et coll. (8) \\
\hline Togo & 19,5 & 10 & & 1,31 & Présente étude \\
\hline \multicolumn{6}{|c|}{ Milieu traditionnel } \\
\hline Côte d'Ivoire & 11,5 & $\begin{array}{l}75 \%<7 \\
11 \%>9\end{array}$ & 206/an & 1,27 & Rombaut et Van Vlaenderen (12) \\
\hline Ghana & 14,3 & 6,95 à 7,8 & & 1,2 & London et coll. (10) \\
\hline Togo & 13 & 7 & & $1,47-1,5$ & Amegee (2) \\
\hline
\end{tabular}




\section{Tableau IV}

Moyennes estimées par la méthode des moindres carrés de l'intervalle entre mises bas des brebis Djallonké élevées au CAT-K

$\begin{array}{lcc}\text { Variables } & \text { Nb. } & \begin{array}{c}\text { Intervalle } \\ \text { entre } \\ \text { agnelages } \\ \text { (j) }\end{array} \\ \text { M oyenne générale } & 263 & 304,0 \\ \text { Année d'agnelage * } & & \\ 1990 & 18 & 268,2 \\ 1991 & 52 & 359,6 \\ 1992 & 73 & 281,5 \\ 1993 & 78 & 334,5 \\ \text { 1994 } & 42 & \\ \text { Saison d'agnelage * } & & 307,4 \\ \text { Pluvieuse } & 241 & 266,5 \\ \text { Sèche } & 22 & \\ \text { Numéro d'agnelage ** } & & 296,7 \\ 1 & 128 & 319,6 \\ 2 & 83 & 296,8 \\ 3 & 52 & \\ \text { Type d'agnelage } & & 292,4 \\ \text { Simple } & 151 & 314,6 \\ \text { Double } & 112 & \\ \end{array}$

$$
\begin{aligned}
& * \mathrm{p}<0,001 \\
& * * \mathrm{p}<0,05
\end{aligned}
$$

L'IMB était influencé par l'année, la saison et le type d'agnelage (tableau IV). L'IMB plus long observé en saison des pluies est en contradiction avec les résultats obtenus au Sénégal par Fall et coll. (8) qui ont rapporté que l'IMB diminuait lorsque la pluviométrie augmentait. Mais chez les bovins et dans les mêmes conditions d'élevage, ces mêmes auteurs (9) ont obtenu un résultat analogue à celui de cette étude. Bien que non significatif, l'IMB était plus long pour les jumeaux que pour les simples.

\section{Taille de la portée, mortalité des agneaux}

La taille de la portée $(1,31 \pm 0,02)$ était proche des résultats de Tuah et Baah (13) mais était supérieure à celle rapportée par d'autres auteurs. Le taux annuel de prolificité (taille portée x IMB/365) était de 1,57 agneau/brebis. Malgré le long IMB observé, il dépassait celui obtenu au CRZ de Kolda (Sénégal) par Fall et coll. (8).

Le taux de mortalité est passé de 1,3 p. 100 à la naissance à 11,7 p. 100 au sevrage (tableau V). La période critique pour la survie des agneaux était le premier mois, au cours duquel près de 10 p. 100 des agneaux sont morts. Bien que relativement élevé, ce taux est inférieur à celui observé au CRZ de Kolda (8) où le sevrage, cependant, se fait à 4 mois d'âge.

Les facteurs ayant eu un effet significatif sur le taux de mortalité présevrage étaient l'année de naissance et le type de naissance. Ainsi, chez les agneaux nés simples qui représentaient 50,8 p. 100 de l'échantillon, la mortalité était de 6,2 p. 100 contre 41,7 p. 100 chez les quadruples dont la proportion n'était cependant que de 0,26 p. 100. S'agissant de l'effet de la saison, Fall et coll. (8) pensent que l'un des principaux facteurs de mortalité des agneaux reste la pluviométrie, la mortalité augmentant de 1,4 p. 100 pour chaque centaine de millimètres de pluie supplémentaires. Toutefois, dans cette étude, bien que la mortalité ait été plus élevée en saison pluvieuse qu'en saison sèche, la différence n'était pas significative.

\section{CONCLUSION}

L'analyse des paramètres de reproduction de la brebis Djallonké au CAT-K montre une relative absence de précocité, un long intervalle entre mises bas, mais une bonne prolificité par rapport aux résultats rapportés par d'autres auteurs chez la même race. Une mise à la reproduction des antenaises dès l'âge de 8 mois et une organisation de saisons de monte de rattrapage devraient permettre d'accroître la productivité numérique des brebis. De même, un meilleur suivi des agnelages et des agneaux (adoption des nés multiples, durée d'une semaine au moins de claustration des femelles après la mise bas) permettrait de réduire la mortalité des jeunes, en particulier, des agneaux nés multiples. Ceci aiderait à réduire au Togo la dépendance de l'extérieur en viande et produits carnés et minimiserait les risques associés à la vache folle.

\section{Tableau V}

Moyennes du taux de mortalité au CAT-K

\begin{tabular}{|c|c|c|c|c|c|}
\hline & N b. & & ux de m & rtalité (c & \\
\hline & & $\mathbf{N}$ & $1^{\mathrm{e}} \mathrm{S}$ & $1^{\mathrm{e}} \mathrm{M}$ & NS \\
\hline $\begin{array}{l}\text { Moyenne } \\
\text { générale }\end{array}$ & 4503 & 1,3 & 6,9 & 9,7 & 11,7 \\
\hline $\begin{array}{l}\text { Année de } \\
\text { naissance }\end{array}$ & & & & & $*$ \\
\hline $\begin{array}{l}1984 \\
1985 \\
1986 \\
1987 \\
1988 \\
1989 \\
1990 \\
1991 \\
1992 \\
1993\end{array}$ & $\begin{array}{r}27 \\
615 \\
365 \\
428 \\
506 \\
679 \\
729 \\
341 \\
340 \\
473\end{array}$ & $\begin{array}{l}0 \\
0,32 \\
0,27 \\
0,70 \\
0,39 \\
1,47 \\
0,96 \\
3,81 \\
1,17 \\
3,15\end{array}$ & $\begin{array}{r}0 \\
5,85 \\
5,75 \\
7,94 \\
5,33 \\
8,83 \\
7,20 \\
10,55 \\
5,00 \\
6,34\end{array}$ & $\begin{array}{r}3,70 \\
9,43 \\
9,36 \\
9,58 \\
8,49 \\
12,07 \\
9,46 \\
14,07 \\
6,47 \\
8,45\end{array}$ & $\begin{array}{r}10,57 \\
11,57 \\
11,51 \\
10,28 \\
10,27 \\
14,14 \\
10,28 \\
21,11 \\
7,65 \\
10,99\end{array}$ \\
\hline $\begin{array}{l}\text { Saison de } \\
\text { naissance }\end{array}$ & & & & & \\
\hline $\begin{array}{l}\text { Pluvieuse } \\
\text { Sèche }\end{array}$ & $\begin{array}{l}3211 \\
1292\end{array}$ & $\begin{array}{l}1,21 \\
1,39\end{array}$ & $\begin{array}{l}6,94 \\
6,73\end{array}$ & $\begin{array}{l}9,68 \\
9,83\end{array}$ & $\begin{array}{l}12,08 \\
10,68\end{array}$ \\
\hline $\begin{array}{l}\text { Type de } \\
\text { naissance }\end{array}$ & & & & & $*$ \\
\hline $\begin{array}{l}\text { Simple } \\
\text { Double } \\
\text { Triple } \\
\text { Q uadruple }\end{array}$ & $\begin{array}{r}2287 \\
2096 \\
108 \\
12\end{array}$ & $\begin{array}{r}0,30 \\
2,14 \\
2,77 \\
16,66\end{array}$ & $\begin{array}{l}3,06 \\
10,2 \\
20,37 \\
33,33\end{array}$ & $\begin{array}{l}4,72 \\
14,02 \\
28,7 \\
41,66\end{array}$ & $\begin{array}{r}6,16 \\
16,51 \\
31,48 \\
41,66\end{array}$ \\
\hline Sexe & & & & & \\
\hline $\begin{array}{l}\text { Femelle } \\
\text { Mâle }\end{array}$ & $\begin{array}{l}2290 \\
2213\end{array}$ & $\begin{array}{l}1,17 \\
1,35\end{array}$ & $\begin{array}{l}6,98 \\
6,77\end{array}$ & $\begin{array}{l}9,52 \\
9,94\end{array}$ & $\begin{array}{l}11,13 \\
12,24\end{array}$ \\
\hline
\end{tabular}

$* \mathrm{p}<0,001$

$\mathrm{N}$ : naissance ; $\mathrm{S}$ : semaine $; \mathrm{M}$ : mois ; $\mathrm{N}-\mathrm{S}$ : naissance-sevrage 


\section{BIBLIO GRAPHIE}

1. ABASSA K.P., PESSINABA J., ADESH O LA-ISHOLA A., 1992. Croissance pré-sevrage des agneaux Djallonké au Centre de Kolokopé (Togo). Revue Elev. Méd. vét. Pays trop., 45 : 49-54.

2. AMEGEE Y., 1983. La prolificité du mouton Djallonké en milieu villageois au Togo. Revue Elev. M éd. vét. Pays trop., 36 : 85-90.

3. BARIL G., CHEMINEAU P., COGNIE Y., GUERIN Y., LEB氏EU B., ORGEUR P., VALLET J.C., 1993. Manuel de formation pour I'insémination artificielle chez les ovins et les caprins. Rome, Italie, FAO, 231 p. (Production et santé animales $n^{\circ} 83$ )

4. BERGER Y., GINISTY L., 1980. Bilan de quatre années d'étude de la race ovine Djallonké en Côte d'Ivoire. Revue Elev. Méd. vét. Pays trop., $33: 71-78$.

5. BOLY H., KOUBAYE A., VIGUIER-MARTINEZ M.C., YENIKOYE A., 1993. Gestion et reprise de l'activité sexuelle après le part chez la brebis Djallonké, varieté « Mossi ». Revue Elev. Méd. vét. Pays trop., 46 : 631-636.

6. CFD, 1996. Relance du secteur de l'élevage dans les pays de la Zone franc après la dévaluation du franc. Paris, France, Ministère de la Coopération Française, $72 \mathrm{p}$.

7. DEVENDRA C., M CLEROY G.B., 1982. Goat and sheep production in the tropics. London , UK, New-York, USA, Longman, $264 \mathrm{p}$.
8. FALL A., DIOP M., SANFO RD J., GUEYE E., WISSO CQ Y.J., DURKIN J., TRAIL J.C.M., 1983. Etude sur la productivité de moutons Djallonké au Centre de recherches zootechniques de Kolda au Sénégal. I. Paramètres de reproduction et viabilité. Revue Elev. Méd. vét. Pays trop., 36 : 183-190

9. FALL A., DIOP M., SANFORD J., WISSOCQ Y.J., DURKIN J., TRAIL J.C.M., 1982. Evaluation of the productivities of Djallonke sheep and $\mathrm{N}$ dama cattle at the Centre de recherches zootechniques, Kolda, Senegal. Addis Abeba, Ethiopia, CIPEA, 74 p. (Research Report N o. 3)

10. LONDON J.C., WENIGER J.H., SCHWARTZ H.J., 1994 Investigations into traditionally managed Djallonke sheep production in the humid and subhumid zones of Asante. Ghana II. Reproductive events and prolificity. J. Anim. Breed. Gen., 11: 432-450.

11. ROMBAUT D., 1980. Comportement du mouton Djallonké en élevage rationnel. Revue Elev. Méd. vét. Pays trop., 33 : 427-439.

12. ROMBAUT D., VAN VLAENDEREN G., 1976. Le mouton Djallonké de Côte d'Ivoire en milieu villageaois. Comportement et alimentation. Revue Elev. Méd. vét. Pays trop., 29 : 157-172.

13. TUAH A.-K., BAAH J., 1985. Reproductive performance, preweaning growth rate and pre-weaning lamb mortality of Djallonke sheep in Ghana. Trop. Anim. Health Prod., 17: 107-113.

14. VALLERAND F., BRANCKAERT R., 1975. La race ovine Djallonké au Cameroun. Potentialités zootechniques, conditions d'élevage, avenir. Revue Elev. Méd. vét. Pays trop., 28 : 523-545.

Reçu le 6.5.97, accepté le 1.4.98

\section{Summary}

Missohou A., Bonfoh B., Kadanga A.K. Djallonke sheep at Kolokopé (Togo): Reproductive traits in ewes and viability of lambs

The aim of this work was to study reproductive performances in 163 Djallonke ewes and viability in 4503 lambs at the Centre d'appui technique of Kolokopé, Togo. Data were collected from 1984 to 1994. Age at first lambing (AFL) and lambing interval (LI) were $595.8 \pm 15.7 \mathrm{~d}$ (19.5 months) and $304 \pm 8.2 \mathrm{~d}$ (10 months), respectively. The litter size and annual reproductive rate were $1.31 \pm 0.02$ and 1.57 lamb per ewe, respectively. The pre-weaning death rate was $11.7 \%$. AFL was significantly influenced by the year of birth $(p<0.001)$, season of birth $(p<0.05)$ and lambing type $(p<0.05)$. LI was significantly affected by the year of lambing $(p<0.001)$, season $(p<0.001)$ and lambing type $(p<0.05)$. The preweaning mortality rate was significantly affected by the year $(p<0.001)$ and birth type $(p<0.001)$.

Key words: Djallonke sheep - Lambing interval - Reproductive performance - Litter size - Birth rate - Mortality - Togo.

\section{Resumen}

Missohou A., Bonfoh B., Kadanga A.K. El ovino Djallonke en Kolopé (Togo): parámetros de reproducción de las ovejas y viabilidad de los corderos

El presente trabajo pretende estudiar los rendimientos de las ovejas y la viabilidad de los corderos D jallonke en el Centro de Apoyo Técnico de Kolokopé (Togo) y se llevó a cabo con 163 ovejas y 4503 corderos. Los datos se recolectaron de 1984 a 1994. La edad al primer parto (EPP) fue de 595,8 $\pm 15,7$ días (19,5 meses), con un intervalo entre partos (IEP) de $304 \pm 8,2$ días (10 meses). El tamaño de la camada y la tasa anual de prolificidad fueron respectivamente de 1,31 $\pm 0,02$ y de 1,57 corderos por oveja. La tasa de mortalidad antes del destete fue de $11,7 \%$. La EPP fue influenciada significativamente por el año $(p<0,001)$ y la estación $(p<0,05)$ de nacimiento y el tipo de parto $(p<0,05)$. En cuanto al IEP, fue afectado significativamente por el año $(p<0,001)$, la estación $(p<0,001)$ y el número de parto $(p<0,05)$. La tasa de mortalidad pre destete fue afectada significativamente por el año $(p<0,001)$ y el tipo de nacimiento $(p<0,001)$.

Palabras clave: O vino - Carnero Djallonke - Intervalo entre partos - Reproductividad - Tamaño de la camada - Tasa de natalidad - M ortalidad - Togo. 